\section{Inclusión curricular de la extensión en el campo de la salud: la vinculación de la universidad con la sociedad desde una nueva perspectiva}

\section{Noemí Bordoni}

Profesora Emérita.

Directora del Instituto de

Investigaciones en Salud Pública.

Universidad de Buenos Aires,

Argentina.

Académica de Número de la

Academia Nacional de Odontología.

nbordoni14@gmail.com
Integración de la docencia y la extensión /

Perspectivas

RECEPCIÓN: 24/06/16

ACEPTACIÓN FINAL: 10/09/16

\section{Resumen}

El marco teórico-metodológico desde el cual se formula este documento plantea que la vinculación se construye articulando las múltiples expresiones y demandas sociales con las formas de producción del conocimiento y las nuevas maneras de organizarlos, distribuirlos e intercambiarlos en los escenarios del equipamiento social. Sus espacios naturales de intervención son aquellos donde se genera el conocimiento y se forman los recursos humanos y en ellos se la programa, ejecuta y evalúa confrontando la teoría en la práctica. En el contexto mundial, la responsabilidad social y cívica de las universidades ha adquirido un protagonismo que la posiciona en la indelegable interacción investigación-docencia-servicio y no como un componente marginal a la investigación y a la docencia. Por lo tanto, analizar la extensión universitaria implica reflexionar sobre la práctica docente, sobre los criterios de calidad y pertinencia que se aplican al evaluar la investigación y sobre el impacto social de sus intervenciones en los escenarios donde opera. El objeto de este informe es analizar, atendiendo a ese marco, los componentes estructurales de la extensión, los procesos de su inclusión curricular y los resultados obtenidos en términos de productos e impacto producido en el campo de la salud en una experiencia desarrollada por la Universidad de Buenos Aires.

\section{Palabras clave}

- Extensión universitaria

- Práctica social curricular

- Articulación docencia-servicio-investigación

- Vinculación universidad-sociedad

\section{Resumo}

O marco teórico-metodológico no qual se baseia este documento propõe que a vinculação se constrói articulando as múltiplas expressões e demandas sociais e as formas de produção do conhecimento e as novas formas de organizá-los, distribui-los e e intercambiá-los nos cenários do equipamento social. Seus espaços naturais de intervenção são aqueles onde se gera o conhecimento e se formam os recursos humanos, e neles é que se programada, executa e avalia confrontando a teoria com a prática. No contexto mundial, a responsabilidade social e cívica das universidades adquiriu um protagonismo que a posiciona na indelegável interação pesquisa-docência-serviço e não como um componente marginal da pesquisa e da docência. Portanto, analisar a extensão universitária implica refletir sobre a prática docente, sobre os critérios de qualidade e pertinência que se utilizam ao avaliar a pesquisa, e sobre o impacto social das suas intervenções nos ccontextos onde opera. O objetivo deste relatório é analisar, atendendo esse cenário, os componentes estruturais da extensão, os processos da sua inclusão curricular e os resultados obtidos em termos de produtos e impacto produzido no campo da saúde em uma experiência desenvolvida pela Universidade de Buenos Aires.

Palavras-chave

- Extensão universitária

- Prática social curricular

- Articulação docência-serviço-pesquisa

- Vinculação universidade-sociedade
Para citación de este artículo

Bordoni, N. (2016). Inclusión curricular de la extensión en el campo de la salud: la vinculación de la universidad con la sociedad desde una nueva perspectiva. En Revista $+E$ versión digital, (6), pp. 50-63. Santa Fe, Argentina: Ediciones UNL. 


$$
\text { N.1. }
$$




\section{Marco teórico-metodológico}

\subsection{Acerca de la conceptualización de salud}

Grandes transformaciones sociales y tecnológicas definen y redefinen constantemente los escenarios donde transcurre la salud e impactan a nivel individual y colectivo. En ese contexto, se han visto afectadas las concepciones sobre los determinantes de los procesos de salud-enfermedad-atención, los métodos de diagnóstico, prevención y tratamiento de las enfermedades, la organización, administración y financiamiento de los servicios y la formación de recursos humanos en salud. Durante mucho tiempo la tradición interpretó la salud en términos de organismos individuales. Una nueva perspectiva concluyó que, para la salud de la gente, el ambiente resultaba más importante que la intervención médica. Esta idea condujo a la aplicación de medidas de salud pública tales como el saneamiento, la vacunación en masa o las campañas diseñadas para modificar conductas no saludables. El modelo de campos de salud identificó a los factores socioeconómicos y a los sistemas de atención asociados con los factores biológicos y el estilo de vida como variables intervinientes en el estado de salud de las poblaciones y fuentes de persistentes inequidades (Laframboise, 1973; Lalonde, 1974; Contandriopoulus, 2006). Glouberman (2006), en el informe "Towards A Dynamic Concept of Health", planteó la hipótesis que admite que la calidad de la interacción entre los individuos y su contexto social es la llave para la salud, en la cual la confianza pública en el sistema sanitario adquiere un significativo impacto sobre la salud junto con otros factores tales como las condiciones biológicas, las relaciones sociales, el estado económico, la experiencia laboral y la cultura. La transición en salud (Frenk,1988) demostró que los cambios demográficos, epidemiológicos, ambientales y de la atención sanitaria, la malnutrición, el insuficiente aporte de agua, la falta de condiciones saludables, las conductas sociales inseguras, el uso del tabaco y de alcohol, los riesgos ocupacionales y la inactividad física son determinantes fundamentales en el proceso de saludenfermedad-atención-cuidados.

En conclusión, la salud en sus diferentes dimensiones y componentes, más que un campo especializado, es un observatorio desde el cual se pueden monitorear muchos de los procesos sociales, económicos y políticos. Es un punto de confluencia donde se articulan la política económica con la social, lo público con lo privado, lo individual con lo colectivo, la equidad

1) Ver Bordoni, 2005a, 2009b y 2010; Birn y Fee, 2013; Murray et al., 2012; con la eficiencia, la reflexión con la acción. Esta interpretación surge de estudios de numerosos autores preocupados por la multidimensionalidad de la salud. ${ }^{1}$

\subsection{Acerca de la institución universitaria}

Existen dos elementos inseparables de la idea de universidad: el conocimiento y los estudiantes. Lemaitre (2003) ha afirmado que no es posible pensar en la universidad sin incluir el compromiso con el conocimiento. Compromiso, que va más allá de la idea de "hacer investigación". Implica la

"conservación y acumulación de esos saberes sobre una base cultural común, el compromiso con el conocimiento teórico y su contrastación con la realidad, con el conocimiento instrumental y su permanente actualización e inclusive con el conocimiento acerca de la institución universitaria, su responsabilidad social y los mecanismos de articulación que es capaz de establecer con la sociedad y el Estado". (Bordoni, 2009b)

La noción de universidad se ha ido reconfigurando en las diferentes épocas. Desde el concepto de universitas aplicado en la Edad Media pasó en el siglo XIX, inclusive por influencia del positivismo, a sufrir un proceso de burocratización (Weber, 1980). Durante el siglo XX, estas instituciones se han mantenido esencialmente idénticas en su estructura y sus métodos y una de las características del mundo de la educación superior ha sido la invariancia de las universidades y su estabilidad en el tiempo (Nuñez, 2002). En general, la universidad acompaña el deslizamiento de todo el espectro social, hecho que se traduce en la categorización de las instituciones de enseñanza y según la cual se privilegian campos de investigación, subsidios y se prestigian publicaciones (Bordoni y Pagliai, 2002).

A partir de la segunda mitad del siglo XX, paralelamente con la producción surgida de los laboratorios de alta sofisticación tecnológica, en los países altamente industrializados se ha generado simultáneamente una revolución silenciosa que determinó un deslizamiento desde los intereses centrados en los estudios de corte econométrico hacia otros intereses, focalizados en la interacción con los "otros" (Inglehart y Bagramian, 2002). En ese marco, las universidades han encarado una revisión de sus propuestas a partir de la reflexión sobre la oferta curricular que realizan, el modelo de práctica al que adhieren y la capacidad para interpretar que la práctica y la formación-docencia-servicio, y logran pertinencia social cuando pueden dar respuestas diversificadas a las necesidades de quienes van a utilizar los conocimientos prácticos impartidos. Este planteo otorga a la extensión una condición estratégica.

Desde la década del '70, en América Latina se desarrollaron proyectos y experiencias universitarias asociados con una marcada 
actividad de la Fundación W. K. Kellogg en el campo de la educación, de la salud y del desarrollo local. Este apoyo estimuló la generación de cambios tendientes a articular las universidades con los servicios de salud y la comunidad y la sensibilización de aquéllas respecto de sus responsabilidades sociales (Bordoni, 1993). En la actualidad se han instalado en el mundo universitario importantes fenómenos. Uno de ellos es la revolución tecnológica, que introdujo desafíos basados en la incorporación de tecnologías de integración, de convergencia y de conexión sobre las representaciones del tiempo y del espacio que determinaron el pasaje desde la tecnología de procesamiento y archivo de la información a la tecnología de conexión. Las relaciones políticas, sociales y culturales se ajustaron a los modelos de red que tienden a reemplazar a las jerarquías tradicionales e instalan relaciones más igualitarias entre sus miembros, entre y dentro de las organizaciones (Castells, 1998 y 2003), inclusive en las universitarias (Tedesco, 1998). La mundialización se instaló y se expresó en sus diferentes interpretaciones — globalización, universalización, internalización, planetarización o aldea global- y en ese contexto hace ya varias décadas que existen universidades sin campus, carreras sin facultades, internacionalización de estudiantes, programas singularizados de acuerdo con las necesidades de la sociedad e institutos virtuales que convocan expertos de diferentes centros o equipos de investigación para abordar problemas en diferentes espacios territoriales (Van Ginkel, 1995; Knight, 2005).

Otro fenómeno está protagonizado por la decisión expresada por parte de la educación superior, a través de sus instituciones o de sus actores, acerca de su intención de participar en la promoción de sociedades más justas y sustentables y definir nuevos perfiles profesionales con esas competencias (Johnston, 1995; Wiltshire, 2001; Bordoni y Mercer, 2004).

La configuración de partenariados entre las universidades, los sistemas de salud (Frenk et al., 2010) y las comunidades que encaran reformas curriculares integrales se reconoce actualmente como una de las estrategias pertinentes para fortalecer la identidad universitaria y reflexionar acerca de:

(a) ¿Cómo debe ser cada facultad y aún cada profesión?

(b) ¿Quiénes son interlocutores válidos?

(c) ¿Cómo facilitar la toma de decisiones?

(d) ¿Cómo trabajar coordinadamente con todos los actores, aun los periféricos, sin desperdiciar las capacidades ni el potencial de desarrollo necesario para avanzar en la direccionalidad requerida por las sociedades?

\subsection{Acerca de la generación de conocimientos}

Para analizar la producción de conocimientos debe identificarse: ¿qué se investiga?, ¿cómo se decide lo que se investiga?, ¿para quién de investiga?, ¿quiénes investigan?, ¿cómo se evalúa a los investigadores? Estas preguntas conducen a reflexionar más allá de los tipos de estudios que se encaran y a la pertinencia metodológica instalada en cada uno de ellos. Las políticas de ciencia y tecnología vigentes en una institución o un país definen los modelos de investigación que se desarrollan (Bordoni y Pagliai, 2002; Gibbons et al., 1993)

En general, los Consejos de Investigaciones se ajustan a diseñar esquemas de organización acordes con los patrones de las disciplinas tradicionales y sobre esa base se asignan recursos y se evalúan investigadores. Esto puede determinar limitaciones en cuanto a resultados socialmente relevantes. Para concretar investigaciones orientadas a problemas se requieren un mayor esfuerzo de gestión y de organización pero constituyen el sustento de un verdadero contrato social entre la ciencia y la sociedad (Abeledo, 2004).

Simultáneamente, uno de los problemas internacionalmente reconocidos es la distancia entre la producción del conocimiento y la toma de decisiones políticas (Bordoni, 2014a; Wiltshire, 2001). Esa preocupación compromete a la profesión en la necesidad de comprender la distancia entre la ciencia y la política y requiere avanzar en el desarrollo de la investigación traslacional, que es un elemento esencial de todo proceso de investigación y un área de investigación por derecho propio (Candiotti de Zan, 2001).

\subsection{Acerca de la formación de los recursos humanos}

Diferentes autores (Camilloni y otros, 1997) plantean que encarar un programa de formación de recursos humanos requiere adoptar previamente grandes decisiones: la elección de la modalidad de organización curricular y de los ejes curriculares ordenadores, la identificación de los criterios de calidad educativa y la formulación del perfil del graduado en términos de competencias efectivas centrales y auxiliares. A partir de esos acuerdos, pueden identificarse los contenidos relevantes ajustados a la mejor evidencia disponible, las experiencias de aprendizaje necesarias para garantizar o favorecer la construcción de conocimientos, inclusive mediante actividades que planteen la ejecución de las mismas tareas en escenarios diversificados o el aprendizaje interactivo con una serie de posibles resultados distintos y, por fin, deben seleccionarse los indicadores que den cuenta del proceso y de los resultados, en términos de productos y del impacto (Bordoni, 2014b).

El currículo se configura al seleccionar y organizar los contenidos de conocimientos en respuesta a la búsqueda de significado para los alumnos, comprobando después de sus respuestas la calidad de "pertinentes" respecto de sus intereses. Consiste en objetos de pensamiento, situaciones, problemas y cuestiones, que existen, aún antes de ser enseñados y que son capaces de desafiar, activar y ampliar las capacidades naturales del ser. Las prácticas en salud, 
como una categoría social construida para dar respuesta a la problemática de la salud y la enfermedad de las personas, existen antes de ser planteadas como objeto de estudio. Esa transposición didáctica (Chevallard, 1999) transita de la práctica a la teoría, del objeto de saber al objeto de enseñanza, de lo implícito a lo explícito. La racionalidad de la acción proviene de la deliberación práctica sobre la forma de ejecutar los fines en función de "actividades realizadas en situaciones complejas concretas" porque operacionalmente los fines se delimitan por la selección de los medios que se utilicen para cumplirlos, de modo que su realización solo ocurre en casos concretos y en circunstancias históricas determinadas (Bruner, 1986; Sacristán y Pérez Gómez, 1989).

\section{La extensión universitaria como objeto de estudio}

\subsection{Acerca de la extensión universitaria ${ }^{2}$}

En América latina, a partir del movimiento universitario iniciado en Córdoba en 1918, la extensión universitaria representó el remate programático de la reforma, con la hipótesis de provocar un acercamiento definitivo entre la universidad y la sociedad, en tanto responsable del sostenimiento de la universidad pública. El reconocimiento de la sociedad y sus instituciones como espacio concreto de intervención universitaria parece haber sido el producto más importante durante los siguientes 50 años. La extensión fue interpretada como un campo destinado al bienestar estudiantil y, eventualmente, a la participación de los docentes o alumnos en actividades reducidas que dieran cuenta de la vertiente benefactora de la institución, sin vinculación con el reconocimiento curricular. Esta interpretación se institucionalizó con las experiencias de voluntariado universitario, aún vigentes en diferentes países e instituciones. El voluntariado, si bien sensibiliza a estudiantes y docentes, en ocasiones carece de los requisitos técnico-científicos adecuados, convirtiéndose en acciones deficientes y sin evaluación y aún, constituyendo intervenciones "para pobres". En otros casos, y como consecuencia de la masificación estudiantil, se convirtió en una herramienta para la desconcentración de estudiantes de grado, fenómeno comprobado en la relación universidad-servicios de salud, determinando la inclusión de estudiantes en el sistema de salud sin que existiera una adecuada garantía de calidad en los criterios diagnósticos, la supervisión docente y las alternativas de tratamientos. Otras perspectivas que se han encontrado han sido: la búsqueda de una oportunidad para optimizar el desempeño estudiantil, la utilización de los estudiantes de grado o posgrado como "mano de obra barata" o la búsqueda de nichos laborales para los graduados. Estas interpretaciones otorgan a la extensión características polisémicas y multidimensionales (Menéndez, 2013). En febrero de 2001, los seniors de las instituciones del más alto nivel pertenecientes a 19 países europeos y norteamericanos, convocados por el Seminario de Salzburgo (2001), se reunieron para intercambiar experiencias e ideas referidas a la misión social y cívica de las universidades, imprimiendo una nueva direccionalidad a las instituciones de educación superior tendiente al fortalecimiento de la interacción y la colaboración entre la vida pública y la académica. Como resultado de las discusiones se acordó sobre la importancia de generar políticas para crear "un escenario para la acción". Esta declaración sustenta la concepción actual de extensión universitaria que, en el campo de la salud, está regida en dos principios básicos: (a) el derecho a la salud como un derecho humano y (b) la responsabilidad social y cívica de la universidad para contribuir a los derechos humanos es tan importante como la investigación y la docencia (Leal, 1995). La extensión resulta de la interacción entre la sociedad con sus múltiples expresiones y demandas para el diagnóstico y la solución de problemas concretos y las universidades, con sus formas de producción del conocimiento y las nuevas maneras de organizarlos, distribuirlos e intercambiarlos en los diversos escenarios del equipamiento social (Bordoni, 2014a).

\subsection{Componentes estructurales de la extensión}

Desde esta perspectiva los componentes requeridos para alcanzar la inclusión curricular de la extensión universitaria son: - La investigación-acción, movimiento basado en el aprendizaje a través de la reflexión, "allí donde tiene lugar la acción" (Kemmis 1988; Elliott, 1989 y 1991), que permite vincular el estudio de los problemas en un contexto determinado, con programas de acción $y$, a la vez, generar conocimientos y traducirlos en cambios sociales. - Las prácticas situadas (Cuadro 1), conceptualizadas de acuerdo con sus diferentes marcos teóricos (prácticas solidarias, aprendizaje-servicio, práctica social curricular, ${ }^{3}$ práctica preprofesional) y con la naturaleza de la contraparte (Cuadro 2), son capaces de determinar un aprendizaje significativo a través del enfrentamiento crítico con la realidad (Henry, 1990; Kolb,1984;
2) Ver Bordoni, 1991, 2005a, 2010 , 2014a; Bordoni y Squassi 2012. 3) Se interpretó como práctica social a las diferentes formas coherentes $y$ complejas de las actividades cooperativas $\mathrm{o}$ integradas pero socialmente establecidas, mediante las cuales los bienes intrínsecos de las acciones se van concretando a medida que se alcanzan los niveles de excelencia que las definen, con la consecuencia de que las potencias humanas para alcan- zar esa excelencia y las concepciones humanas de los fines y bienes implicados se amplían de modo sistemático. 
Chaiklin y Lave, 2001; Lucarelli, 2004; Camilloni, 2013).

- La planificación estratégica como herramienta para realizar el análisis de la situación, la reflexión y la toma de decisiones para direccionar acciones presentes y futuras, con respuestas a cambios y demandas del contexto sin perder su identidad y garantía de calidad en sus intervenciones.

- La participación social que permite intervenir en la toma de decisiones a quienes tienen que ver con el proyecto, introducir en

\section{Cuadro 1}

Prácticas situadas en la comunidad en el campo de la salud

Son una forma de dar relevancia en el nivel educacional a las necesidades de la comunidad.

Las actividades se desarrollan un entorno educacional compuesto por estudiantes, docentes, miembros de diferentes sectores de la comunidad y el sistema de salud.

El equipo de salud forma parte de la comunidad.

El diagnóstico de necesidades hecho por y con la comunidad determina el perfil de los profesionales que van a trabajar en ella.

Condicionan el diseño de los programas de pre y posgrado y el desarrollo de diferentes estrategias de enseñanza y de aprendizaje.

Contribuyen a solucionar la inequidad en salud, producen recursos humanos con un perfil pertinente.

Refuerzan el proceso de aprendizaje de modo similar al Aprendizaje Basado en Problemas.

Proveen a los profesionales y estudiantes de herramientas que de otro modo no hubieran adquirido: trabajo en equipo, liderazgo, capacidad de interactuar con toda la comunidad.

Ofrecen la posibilidad de aprender y formarse junto a profesionales de otras ramas en un ámbito multiprofesional.

Dado que se basa en el cuidado de la salud, el currículo es dinámico.

Ofrecen la posibilidad de unir esfuerzos entre la comunidad, la universidad, ONG, el sector privado y el gobierno.

\section{Cuadro 2}

\begin{tabular}{|c|c|c|}
\hline \multicolumn{3}{|c|}{ Metodologías universitarias desarrolladas en la extensión } \\
\hline \multirow[t]{2}{*}{ Metodologías universitarias en la parcería } & \multicolumn{2}{|l|}{ Marco jurídico-administrativo de la vinculación } \\
\hline & $\begin{array}{l}\text { Estrategias de la vinculación con organizaciones } \\
\text { sin fines de lucro }\end{array}$ & $\begin{array}{l}\text { Estrategias de la vinculación con organizaciones } \\
\text { con fines de lucro }\end{array}$ \\
\hline \multirow[t]{2}{*}{ Cooperación técnica estratégica (cooperar con...) } & \multirow[t]{2}{*}{ Práctica social curricular y/o redes estratégicas } & Pasantías supervisadas \\
\hline & & Proyectos de desarrollo local \\
\hline \multirow[t]{3}{*}{ Asistencia técnica institucional (asistir a...) } & Informes técnicos (expertos nacionales/internacionales) & Informes técnicos (expertos nacionales/internacionales) \\
\hline & Formación de recursos humanos & Formación de recursos humanos \\
\hline & Capacitación permanente & Capacitación no sistemática \\
\hline \multirow[t]{2}{*}{ Servicios directos sobre (intervenir sobre...) } & Servicios universales & Grupos bajo cobertura contractual \\
\hline & Servicios a grupos vulnerables & Seguros de salud/enfermedad \\
\hline
\end{tabular}

el debate las cuestiones pertinentes, establecer nuevas alianzas y mejorar la identificación local del proyecto (Fidler, 2009; Saltman et al., 2011).

- La gestión integrada, entendida como una asociación entre actores diferentes para el logro de fines comunes, donde los vínculos se dan en pie de igualdad o semejanza, se superan los poderes relativos de las partes, se tiende al mejoramiento de cada parte y del conjunto.

Sin embargo, los cambios instalados por la sociedad-red (Nowotny et al., 2001; Castells, 2003) y por la interdisciplinariedad como práctica social que en la cual la complejidad deriva no solo de la heterogeneidad sino de la interdefinibilidad y de la mutua dependencia de las funciones que cumplen esos elementos dentro del sistema total (García, 2006), han generado deslizamientos sobre estos componentes. Se produjo un tránsito:

- Desde la investigación-acción a la investigación traslacional, que toma como objeto de estudio la construcción social de un conocimiento preconstruido (Bordoni, 2014a).

- Del aprendizaje en escenarios reales hacia la generación de aprendizaje significativo y relevante para el alumno, tendiente a "hacer y reflexionar sobre la propia práctica" (metacognición) y que explora el impacto social de las intervenciones mediante indicadores indirectos.

- Desde la participación comunitaria a la gobernanza en la toma de decisiones mediante la práctica permanente de procesos deliberativos interactivos (Natera Peral, 2004; Saltman et al., 2011). - Desde la planificación estratégica y la asistencia técnica, a la cooperación técnica estratégica, trabajando con los "otros" los momentos analíticos, normativos y tácticos planificados cooperativamente para la construcción de redes o alianzas estratégicas (Rovere, 1999).

- Mediante la interpretación de la gestión integrada como un instrumento de democratización. 


\section{La universidad en acto: gestión de la extensión. Acerca del desarrollo institucional}

En 1979 se incorporó al currículo de la carrera de Odontología la Unidad Funcional de Apoyo Docente Asistencial incluyendo docentes investigadores formados en la escuela odontopediátrica argentina y con marcada influencia técnica de la escuela escandinava. Esta cátedra era la responsable de la enseñanza y el aprendizaje de la prevención, la educación para la salud y la metodología científica a lo largo de los cinco años de la carrera. En el área clínica se concretó la transferencia mediante la formulación de protocolos de atención con fuerte componente preventivo que permanentemente fueron y son actualizados (Bordoni, 1983).

Sin embargo, la eficacia de esta instancia de formación fue limitada debido a que proponía un nuevo modelo de atención clínica pero mantenía una visión incompleta del sistema y de los servicios de salud y de otras formas de la práctica odontológica que se concretaban en "el afuera" de la institución. Por lo tanto, el proceso transitado hasta 1984 había sido necesario pero no era suficiente (Bordoni, 2005a).

En 1985, coincidente con la normalización universitaria, se realizó un diagnóstico curricular con apoyo internacional y asesoramiento pedagógico tendiente a encarar un cambio en la carrera de odontología de la UBA. Los cambios más importantes fueron:

a) La elección del eje curricular que incorporara: las clínicas integradas (1984-1998), la departamentalización con relación básico-clínica (1984-1996), ${ }^{4}$ y la Articulación Docente Asistencial (ADSI), ${ }^{5}$ vigente en el currículo actual, y el establecimiento de convenios con el gobierno de la Ciudad Autónoma de Buenos Aires y otros municipios para su articulación con hospitales y escuelas (Bordoni, 1993; 2009a).

b) El fortalecimiento de la investigación y la creación del Instituto de Investigaciones Odontológicas (1984-1990).

c) La conceptualización del proceso de aprendizaje como construcción social y el desplazamiento de la centralidad del proceso de enseñanza hacia la comunidad, el paciente y el alumno.

\subsection{Acerca de la extensión curricular en la Facultad de Odontología (FOUBA)}

La inclusión curricular de la extensión se inicia en la FOUBA como parte del cambio de 1985, con la articulación docenciaservicio-investigación (ADSI) en la comunidad planteada como una estrategia de trabajo mediante la cual los organismos administradores de servicios de salud y las instituciones formadoras se vinculan con dicha comunidad y utilizan los recursos existentes para la planificación, ejecución y evaluación de las acciones de salud a fin de elevar la calidad en sus diversas expresiones y contribuir al mejoramiento de las condiciones de salud de la población (Bordoni, 1993). Desde su incorporación pueden identificarse tres etapas, no necesariamente secuenciales, e inclusive recurrentes o simultáneas.

La cobertura registrada en las sedes urbanas, entre 1985 y 2003 , alcanzó a 133626 personas y a 22345 en las sedes rurales o urbano-marginales intensivas con acreditación curricular.

\subsection{Desarrollo de la Primera Etapa: Práctica social curricular} La Primera Etapa constituyó la instalación de la Práctica social curricular de naturaleza disciplinar, que transitó tres momentos. El primer momento encaró el crecimiento endogámico con los siguientes alcances:

- Definiciones conceptuales y protocolización de la práctica clínica individual basada en el enfoque de riesgo (Bordoni, 1981, 1983, 1997).

- Consolidación de los equipos docentes.

- Producción de investigaciones y tesis de doctorado.

- Obtención de subsidios nacionales e internacionales, entre ellos, el Proyecto Articulación Docencia-Asistencia-Investigación (PADAI) subsidiado por la Fundación W. K. Kellogg entre 1985 y 1992 (Bordoni, 1991; 1993, 1994) y posteriormente los proyectos Redes y Norte-Sur.

El segundo momento apuntó al desarrollo de investigaciones respecto de la eficacia y efectividad de los programas incluso de costo/eficacia y costo/efectividad (Doño y otros, 1983, Bordoni cente Asistencia dio lugar a la cátedra de Odontología Preventiva y Comunitaria, que asumió la responsabilidad curricular de la extensión. 


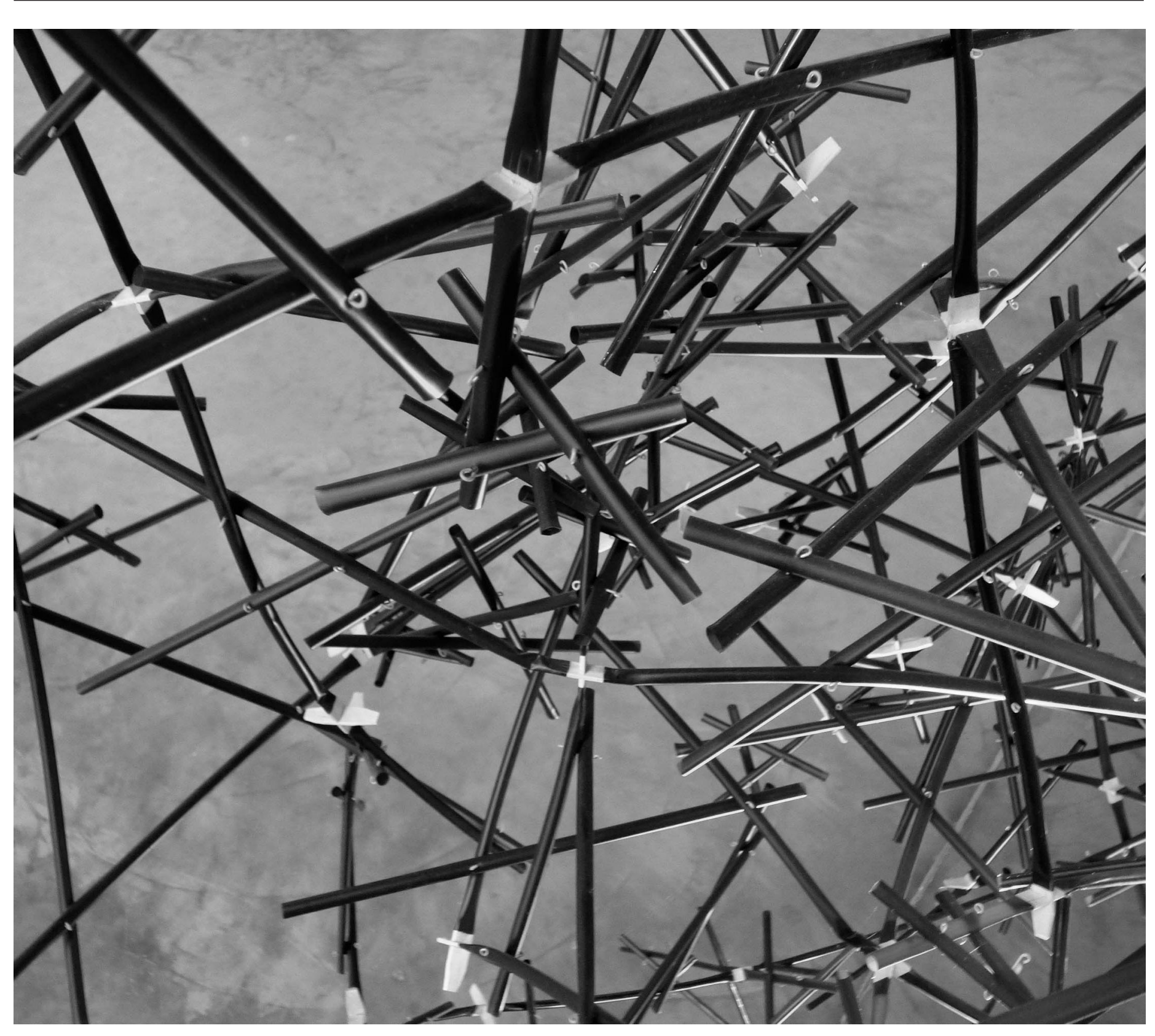

() Micaela Block 
et al., 2005; Bordoni y Squassi 2013;) y fundamentalmente a la definición conceptual referida a la vinculación con organizaciones gubernamentales (Bordoni 2009a) y no gubernamentales. Tal es el caso de:

- La Práctica social curricular, diferenciada de voluntariados y de prácticas preprofesionales.

- Los distintos modos de vinculación (cooperación, coordinación o integración).

- Los diversos planos operacionales (individuales, familiares, regionales o globales).

El tercer momento se caracterizó por la diseminación de la propuesta; se desarrollaron cinco cursos de alcance internacional, tales como el Programa de Educación Odontológica no Convencional (PRECONC) (Bordoni et al.; 1999; Bonazzi et al., 1997) incorporado al PALTEX (Programa de Libros de Texto) de OPS/OMS, con 19000 libros publicados y agotados y más de 9000 profesionales evaluados con responsabilidad de la Odontología Preventiva y Comunitaria / FOUBA y gestionado por docentes entrenados pertenecientes a otras universidades de Argentina, México, Uruguay, Chile, Perú, Ecuador, Venezuela, República Dominicana, Panamá, Costa Rica, El Salvador, entre otros países (Bordoni, 1991 y 1997). Otra experiencia docente con trabajo en campo fue el curso latinoamericano "Componente bucal de la Atención Primaria de la Salud", que contó con cursantes de España, Cuba, Chile, Uruguay, Paraguay, Colombia, Perú, Brasil, México y, posteriormente, la Maestría de Odontología Comunitaria hasta 1996.

\subsubsection{Sedes de intervención iniciadas durante esta etapa} Las sedes incorporadas al proceso de extensión curricular con vinculación formal entre la UBA y la contraparte durante ese lapso fueron:

- Hospitales y centros de salud dependientes del Gobierno de la Ciudad Autónoma de Buenos Aires (CABA) y de seis municipios del Gran Buenos Aires.

- Escuelas dependientes del Gobierno de la CABA y de cuatro municipios del Gran Buenos Aires.

- Dos ONG.

- Comunidades de las provincias de Misiones y La Rioja y dos barrios del Área Metropolitana de Buenos Aires.

\subsection{Desarrollo de la Segunda Etapa: Redes interdisciplinarias} La etapa "Redes interdisciplinarias" se introdujo entre los años 1994-1996. Se inició con el desarrollo de la Maestría en Salud Pública dependiente del Rectorado de la UBA con participación de 13 Facultades de la Universidad (Cadile y otros, 2006). En el año 2007 se diseñó e implementó un Instituto de Investigaciones en Salud Pública dependiente de la UBA, a través de la Secretaría de Ciencia y Técnica, configurado por equipos interdisciplinarios adheridos convocados para la resolución de problemas de salud complejos existentes en diversas bases territoriales.

El proyecto UBACYT R 001 "Estudio sobre problemas prevalentes y emergentes en escolares y familias residentes en áreas con riesgo social" constituyó un ejemplo de redes interdisciplinarias en la cuales participaron equipos de las siguientes facultades: Odontología, Ciencias Exactas y Naturales, Farmacia y Bioquímica y Agronomía.

\subsubsection{Resultados obtenidos durante esta etapa}

- Diseño de un modelo de instituto virtual para desarrollar investigación por problemas, y aprobación por la Universidad de Buenos Aires (Res. Rector 584/07 y Res. CS 2231/07).

- Trabajos de campo enfocados en cuatro categorías: (a) problemas de salud específicos, (b) análisis evaluativos de programas o proyectos; (c) análisis evaluativos de sistemas o servicios (Pagliai, Bordoni; 2007); (d) situación de salud en bases territoriales.

- Diseño y validación de instrumentos para la evaluación de la calidad de vida (Squassi et al.; 2006; 2010)

- Diseño y validación de instrumentos para la determinación del impacto social (Bordoni y Squassi, 2011).

- Publicaciones de trabajos científicos y documentos técnicos.

\subsubsection{Sedes de intervención}

Comunidades de la provincia de Chaco (Facultad de Ciencias

Exactas y Naturales e Instituto de Investigaciones en Salud Pública de la UBA).

Comunidades de la provincia de Misiones (Facultades de Odontología, Agronomía, Farmacia y Bioquímica, Ciencias Exactas y Naturales e Instituto de Investigaciones en Salud Pública, UBA. Villa $11 y 14$ de la CABA (FCEyN e IISAP/UBA).

Programa escolar de la CABA y Municipios del Área metropolitana (Facultad de Odontología, UBA).

\subsection{Desarrollo de la Tercera Etapa. Proceso traslacional ${ }^{6}$} La Tercera Etapa encarada por el Instituto de Investigaciones en Salud Pública de la UBA con la participación de los equipos asociados de las diferentes facultades se fue consolidando especialmente a partir de 2010.

La investigación traslacional consiste en tomar como objeto de estudio el trabajo de construcción social de conocimientos preconstruidos. Se considera un componente esencial del proceso de investigación y un campo de investigación por derecho propio, puede desarrollarse en diferentes escenarios e incluir actores externos a la investigación. Implica transitar dos momentos. - Momento 1: Generación de procesos deliberativos interactivos (PDIs). 
Un PDI es un proceso que permite a un grupo de actores recibir e intercambiar información, examinar críticamente los asuntos y arribar a un acuerdo que sustente la toma de decisiones (Saltman et al., 2011). Pueden ser: operacionales (maestros, padres de familia), colectivos (directores de escuelas, jefes de servicios) o constitucionales (secretarios de Salud, ministros).

- Momento 2: Inversión del proceso tradicional de traslación desde la producción de conocimientos a la toma de decisiones políticosanitarias (Bordoni 2014a).

Incluye la sucesión de actividades siguientes:

(a) Producción de conocimientos, resultado de la investigación básica (sistema cerrado con debate entre pares, variables controladas y evaluación de estructura y productos).

(b) Introducción de PDIs preferentemente multinivel y eventualmente multicéntricos que tiendan a un amplio debate de los problemas en busca de acuerdos eventuales respecto de las decisiones a adoptar. (c) Construcción de nuevos objetos de estudio sobre determinantes, nuevas problemáticas complejas y soluciones pertinentes basadas en la mejor evidencia disponible (abordaje multidisciplinario).

(d) Desarrollo de investigaciones interdisciplinarias desarrolladas en contextos de incertidumbre, con triangulación de resultados y evaluación de los procesos y del impacto social.

(e) Mantenimiento de PDls y eventual consolidación de modelos de gobernanza.

(f) Toma de decisiones sobre las problemáticas priorizadas e instalación en la agenda política (sistema abierto e intersectorial).

(g) Evaluación de las intervenciones en términos de resultados (productos e impacto social).

(h) Protocolización, difusión, capacitación y diseminación para la incorporación en la clínica y la evaluación y la obtención de evidencia contextualizada.

(i) Inducción de nuevas investigaciones.

\subsubsection{Ejemplos de decisiones político-sanitarias basadas en la vinculación universidad-sistema de educación}

La cátedra de Odontología Preventiva y Comunitaria de la Facultad de Odontología de la UBA incorporó la modalidad de cooperación técnica estratégica con el Ministerio de Educación del Gobierno de la $\mathrm{CABA}$, desarrollando programas preventivos escolares de primer nivel de atención con participación de profesionales docentes. En el proyecto UBACYT /R 022: "Salud global: estudio sobre determinantes, procesos deliberativos e impacto social" y en otras acciones de la articulación docencia-servicio-investigación se pusieron en acto la construcción de redes estratégicas y el modelo de traslación a decisiones político-sanitarias. Los problemas de salud priorizados que convocaron el inicio del proyecto fueron: las enfermedades bucales prevalentes y emergentes, las enteroparasitosis y la toxocariosis, las enfermedades vinculadas con el uso de agroquímicos y la problemáticas de atención de salud en grupos originarios.

\section{Resultados en un área modelo de demostración (1984-2015)} La base territorial inicial fue la Municipalidad de Caraguatay, provincia de Misiones. La problemática priorizada al inicio del programa fue el deficiente estado bucodental en escolares, mientras que la intervención programada incluyó actividades basadas en la evidencia científica contextualizada. Las actividades anuales se desarrollaron y desarrollan aún con participación de alrededor de 50 estudiantes de sexto año de la carrera de Odontología y 15 docentes durante una semana con dedicación intensiva.

\section{Conclusiones}

1. En la FOUBA, la extensión universitaria tiene carácter curricular en el nivel de grado desde 1985 incorporado en el plan de estudios en las asignaturas Articulación Docencia-AsistenciaInvestigación I y II (1985-1999) o Articulación Docencia-Servicios de Salud (2000 y continúa) y como asignaturas optativas con crédito curricular en los programas rurales respondiendo al modelo Práctica social curricular.

2. La vinculación entre FOUBA y organismos gubernamentales dependientes del Ministerio de Educación del Gobierno de la CABA adopta la modalidad cooperación técnica estratégica con participación de profesionales docentes.

3. La extensión universitaria desarrollada entre la FOUBA, la FCEyN, la FA, la FFyB y el IISAP en el marco de la programación UBACyT se encuentra focalizada en actividades de investigación clínicoepidemiológica o traslacional con diferentes alcances de impacto. 
- Conceptualización de la Práctica social curricular como estrategia de vinculación entre universidad y organizaciones sin fines de lucro (Bordoni, 1993; 2014a y b).

- Cobertura atención bucodental anual media estimada: 450 escolares y 150 adultos.

- Reconversión de problemas bucodentales prevalentes, prevenibles y evitables en niños asistentes a escuelas 168 y 467 (departamento de Caraguatay).

- Capacitación de becarios internacionales asistentes al programa (Chile, Uruguay, México, Ecuador, Colombia, España, Costa Rica, Cuba, Paraguay).

1995-1998

\section{9-2003}

Nuevos problemas, nuevas intervenciones: Identificación de nuevos objetos de estudio sobre problemáticas complejas a partir de estudios multidisciplinarios: embarazo adolescente, hábitat insalubre, calidad del agua, contaminación por agroquímicos, problemas neurológicos de diferente etiología, otros problemas bucodentales. obtenidos mediante la evaluación de estructura e impacto social

mediante evaluación de estructura e impacto social
- Sistematización y análisis de los componentes de la PSC: (a) investigación-acción, (b) planificación estratégica, (c) participación social, (d) prácticas situadas y (e) gestión integrada.

- Ampliación de la cobertura en comunidades del departamento de Caraguatay en términos territoriales (Taruma y Mato Quemado) y de niveles etarios (preescolares y adolescentes y adultos).

- Cobertura atención bucodental anual estimada: media de 1100 niños y 400 adolescentes y adultos.

- Reconversión del perfil de problemas bucodentales prevalentes, prevenibles y evitables en niños asistentes a escuelas 168 y 467 (departamento Caraguatay).

- Análisis de situación en las áreas rurales dispersas para el desarrollo de equipamiento en las aulas satélites.

- Ampliación de la cobertura en términos de niveles etarios preescolares y adolescentes y adultos) y de áreas rurales dispersas cubiertas (aulas satélites).

- Cobertura atención bucodental anual estimada: 1200 niños y 400 adolescentes/adultos.

- Reconversión significativa de problemas bucodentales prevalentes, prevenibles y evitables en grupos de diferentes edades.

- Solicitud de la comunidad de padres para instalar una sede de atención en la Escuela n 426 de Taruma.

- Organización de familias y docentes locales en diferentes comunidades del departamento de Caraguatay (Mato Quemado) para la participación social y la gestión integrada de recursos humanos (participación de maestros y padres) y de recursos físicos (preparación de camillas y bancos destinados a operadores y ayudantes, de acuerdo con criterios ergonómicos por parte de la comunidad local.

\section{4-2007}

obtenidos
mediante
evaluación de
estructura e
impacto social

- Cobertura atención bucodental anual estimada: 1350 niños y 500 adolescentes y adultos.

- Obtención de proyectos UBANEX/UBA por OPyC de FOUBA y por el IISAP/UBA.

- Formalización de Convenio UBA/Municipalidad Caraguatay.

- Incorporación de Escuela Agraria al programa rural.

- Ampliación de la cobertura a las escuelas y familias del departamento de Montecarlo.

- Vinculación de FOUBA con equipos IISAP/UBA para abordar estudios interdisciplinarios.

- Análisis de situación de salud por parte del equipo del Departamento de Química Biológica de la Facultad de Ciencias Exactas y Naturales/UBA adscripto al IISAP/UBA. 


\section{8-2015}

Nuevos problemas, nuevas intervenciones ${ }^{7}$

- Desarrollo de investigaciones

interdisciplinarias con evidencia científica en contextos de incertidumbre, triangulación de resultados y evaluación de procesos e impacto social.

- Introducción de la cátedra de Sociología Rural (Facultad de Agronomía/UBA adscripto IISAP/UBA) desde 2009 para investigaciones del uso de agroquímicos y su impacto sobre la salud (Souza, 2013). - Mantenimiento de los PDIs y consolidación de modelos de gobernanza tendientes a elevar propuestas concretas aceptadas por todos los actores sociales para la toma de decisiones político-sanitarias.

\section{obtenidos}

mediante

evaluación de

estructura e

impacto social

(OPyC e IISAP)

UBA)

del trabajo

interdiciplinar

entre Sociología

rural con la

coordinación

de Odontología

Preventiva y

Comunitaria

e Instituto de

vestigaciones

en Salud

Pública/UBA
- Obtención de Proyectos UBACYT del IISAP/UBA 2011-2014 (aprobado).

- Obtención de Proyectos UBACYT del IISAP/UBA 2014-2017 (en desarrollo) con base territorial

en la región.

- Obtención de Proyectos UBANEX por Odontología Preventiva y Comunitaria (Facultad de

Odontología, UBA) y de la Facultad de Ciencias Exactas y Naturales de la UBA.

- Cobertura de la atención bucodental anual estimada: 2100 niños y 680 adolescentes /adultos.

- Profundización de la cobertura en salud bucal diagnosticando maloclusiones (método ICON).

- Los Procesos Deliberativos desarrollados en el departamento de Caraguatay (Paraje El Lavarropas y escuelas) durante 2009 y 2011 permitieron la interacción con 140 miembros de la comunidad capacitados con información sobre el efecto de los plaguicidas sobre la salud.

- Desarrollo de investigaciones acerca de contaminación por agroquímicos usados en la zona.

- Los PDIs desarrollados en el departamento de Montecarlo permitieron la interacción con las familias semilleras de la Feria franca y con productores agrícolas y la capacitación a docentes en la Escuela Especial $n^{\circ} 1$ de Montecarlo.

- Se realizaron entrevistas con miembros del Concejo Deliberante, intendentes y concejales (Procesos Deliberativos Interactivos de tipo constitucionales) y con los medios de comunicación - En 2015 se visitaron medios productivos; se concretaron: tres instancias de capacitación con productores y escuelas, tres reuniones con tomadores de decisión y cuatro entrevistas con los medios de comunicación.

- En total, la vinculación alcanzó a más de 190 personas capacitadas o informadas y a 10 participaciones en los medios de comunicación.

- El Concejo Deliberante de la Municipalidad de Montecarlo prohibió el uso de plaguicidas en zonas urbanas y reguló su uso en las colonias, limitando la distancia mínima a mantener hasta las casas y arroyos. Está en vigencia (2016).

del trabajo

- Investigaciones del Departamento de Química Biológica (Facultad de Ciencias Exactas y

interdisciplinar

entre Química

Biológica,

coordinado por

Odontología

Preventiva y

Comunitaria

e Instituto de

investigaciones

en Salud

Pública/UBA
Naturales/ UBA). Desde 2008 se tradujeron en tesis y publicaciones sobre enteroparasitosis y métodos e instrumentos educativos para diagnóstico y prevención familiar.

- El impacto producido se encuentra en el punto (d) (Desarrollo de investigaciones interdisciplinarias con evidencia científica en contextos de incertidumbre, triangulación de resultados y evaluación de procesos e impacto social (Buyayisqui et al., 2013; Garbossa et al., 2013).

7) En el año 2016 se ha incorporado para nuevas investigaciones la FFyB de la UBA (Equipo de cátedra de Toxicología). 


\section{Referencias bibliográficas}

Abeledo C. (2004). Investigación orientada a la solución de problemas. En Pugliese J. C. Universidad, Sociedad y Producción. Buenos Aires: MECyT.

Birn, A.; Fee, E. (2013). The Rockefeller Foundation and the international health agenda. The Lancet, 381, 1618-1689.

Bonazzi, M.; Squassi, A.; Bordoni, N. (1997). Distance Education Program: Analysis of intervening variables. J Dent Res, 76, 345 (Abs.).

Bordoni, N. (1981). Programas para la atención clínica preventiva a nivel individual. Rev. Asoc. Odont. Arg., 69 (2), 71-85. Disponible en portugués (Programas de atencao clinica preventiva a nivel individual, Quintaesséncia IV Rel 0856, 9, 1-21 (Port) Anuario de Quintaessence.

Bordoni, N. (1983). Programas preventivos. Propuesta para racionalizar la atención clínica. Rev. Asoc. Odont. Arg., 71, 212-220.

Bordoni, N. (1991). Papel de la Universidad en el sistema de prestación de servicios. En Actas de la Reunión Norte-Sur: "Avances tecnológicos y su impacto en la educación odontológica en el siglo XXI". Puerto Rico: Universidad de Puerto Rico. Bordoni, N. (1993). Modelo alternativo para la atención en Salud Bucal Documento para la transferencia tecnológica. Buenos Aires: Fundación W: Kellogg/PADAlFOUBA/OFEDU-UDUAL.

Bordoni, N. (1994). Non conventional Continuing Dental Education Program. Human Resources in Oral Health. Puerto Rico: University of Puerto Rico/AADSOFEDO/UDUAL.

Bordoni, N. (1997). Programas preventivos según enfoque de riesgo. En Uribe Echeverría, J. y Fernández Bodereau, E. (Eds.), Odontología clínica a fines del milenio. Córdoba: Universidad Nacional de Córdoba.

Bordoni, N. (2005a). Vinculación entre la sociedad y la universidad. En Pugliese, J.C. Universidad, Sociedad y Producción. Buenos Aires: MECyT.

Bordoni, N. (2009a). Estudio de un caso de integración entre la investigación, la docencia y la extensión en el escenario de la atención odontológica. En Riquelme, G. C. (Ed.), Sinergia pedagógica en universidades argentina: articulación de la docencia, la investigación, la extensión y la transferencia en el desarrollo de planes de estudio. Tomo III. Buenos Aires: Miño y Dávila Editores.

Bordoni, N. (2009b) Nueva mirada sobre la formación de recursos humanos en Odontología. Revista Encrucijadas UBA, 47, 35-39.

Bordoni, N. (2014a). Programas interdisciplinarios: Una mirada desde los problemas y "Proyectos universitarios para la mitigación de la marginación social. Perspectiva de los investigadores." En Secretaría de Ciencia y técnica. UBA: Universidad y Sociedad. Desafíos de la investigación interdisciplinaria. Buenos Aires. Eudeba.

Bordoni, N. (2014b). Formación de recursos humanos en salud para el desarrollo. Medicina y sociedad. Julio. Disponible en http://www.medicina y sociedad.org.ar/ Bordoni, N. et al. (1992, 1998, 1999). Programa de Educación Odontológica no Convencional PRECONC/PALTEX - OPS/OMS. Tomo 1 (3ª. ed. 1999); Tomos 2, 3 y 4 (1 $1^{\text {a }}$ ed.1992); Tomo 5 ( $1^{\text {a }}$ ed. 1998).

Bordoni N; Pagliai L. (2002). Assessment Of Health Research Systems: Critical Review Of Pragmatic Agreement Current Indicators. Document present in the Session of Council of Health Research for Development. (COHRED/WHO) Paris, October . Disponible En: Revista de Salud Pública de la UBA. (on line). 2003; Vol I, Agosto revista.maestria.rec.uba.ar
Bordoni N; Mercer H. (2004). Vinculación de la universidad con la sociedad. En: Borrell RM (Ed) Los posgrados de Salud Pública. Buenos Aires, OPS/OMS.

Bordoni, N.; Squassi, A; Bellagamba, H; Galarza, M. (2005). Efficiency of a schoolchildren program for oral care. Acta Odont Latinoamericana, 18 (2), 75-81.

Bordoni N; Squassi A. (2011). Diseño de un instrumento para la evaluación del impacto de las investigaciones en salud. Reunión IADR/Div Argentina (Abs.). Bordoni N; Squassi A. (2012). Vinculación entre la universidad y la sociedad: experiencias de la Universidad de Buenos Aires. En Sánchez Medina, J., López Vidales, R. (Eds.), Promoción de salud escolar en Iberoamérica: Experiencias. Documentos. Vol. 1. Sevilla: Red Iberoamericana de Promoción de Salud Escolar (RIPSE).

Bordoni, N.; Squassi, A. (2013). Caries dental: una mirada actual para una vieja problemática/Dental caries: a new Outlook to an old problem. Revista Universitas Odontológica, 32(68), 81-97. Pontificia Universidad Javeriana.

Bordoni, N., Escobar, A., Castillo, R. (2010). Odontología Pediátrica. La salud bucal del niño y el adolescente en el mundo actual. Buenos Aires: Ed. Médica Panamericana. Capítulos 6, 43, 46, 48 y 50.

Borrell, C.; Malmusi, W. (2010). Investigación y determinantes sociales de salud e inequidades de salud: evidencia en todas las políticas. Gac. Sanit., 24 (Supp1), $101-108$.

Braverman, P.; Gruskin, S. (2003). Defining equity in health. J Epidemiol Community Health, 57, 254-258.

Breilh, J. (2010). La epidemiología crítica: una nueva forma de mirar la salud en el espacio urbano. Salud colectiva, 6(1), 1-19.

Bruner, J. (1986). Realidad mental y mundos posibles. Los actos de imaginación que dan sentido a la experiencia. Barcelona: Gedisa.

Bruner, J. (1997). La educación puerta de la cultura. Madrid: Visor.

Buyayisqui, M.P.; Bordoni, N.; Garbossa, G. (2013). Overcoming Language and Cultural Barriers. A Graphical Communication Tool to Perform a Parasitological Screening in two Vulnerable Populations from Argentina. Journal of Health Communication, 18(1):92-104. Disponible en: https://www.ncbi.nlm.nih.gov/ pubmed/23066862\# doi: 10.1080/10810730.2012.688242.

Cadile, M.; Aulicino, M.; Duré, I.; Bordoni, N. (2006). Vinculación entre la Universidad y el Sistema de Salud. Un modelo en desarrollo. Revista Maestría Salud Pública UBA, 6 (12). Disponible en: http://msp.rec.uba.ar/revista/index.php Camilloni A. (2013). La inclusión de la educación experiencial en el curriculum universitario. En Menéndez G. y otros, Integración docencia y extensión. Otra forma de enseñar y aprender. Santa Fe: Ediciones UNL.

Camilloni, A.; Davini, M. C. y otros (1997). Corrientes didácticas contemporáneas. Buenos Aires: Paidós.

Candiotti de De Zan, M.E. (2001). La construcción social del conocimiento. Aportes para una concepción crítica del aprendizaje. Buenos Aires: Santillana.

Castells, M. (1998). La era de la información. Economía, sociedad y cultura. Madrid: Alianza.

Castells, M. (2003). The rise of the nework society. (2d. ed). USA: Blackwell Pub.

Chaiklin, S.; Lave, J. (2001). Estudiar las prácticas: Perspectivas sobre actividad y contexto. Buenos Aires: Amorrortu.

Chevallard, Y. (1999). La transposición didáctica. Del saber sabio al saber enseñado. Madrid: Aiqué.

Contandriopoulos, A. P. (2006). Elementos para una "Topografía" del concepto de 
salud. Ruptures, Revista Interdisciplinaria de la Salud, 11(1), 86-99. Universidad de Montreal. Canadá

Doño, R.; Cantón, L.; Argentieri, A. (1983). Estudios de costo-eficacia de programas preventivos escolares. Rev. Asoc. Odont. Arg., 71, 223-231.

Elliott, J. (1989). Teacher evaluation and teaching as a moral science. En Holly, M.L.; Mc Loughlin, S. (Eds.), Perspectives on Teacher Professional Development. London and New York: Palmer Press.

Elliott, J. (1991). Action-research for educational change. London: Open University Press.

Fidler, D. P. (2009). After the revolution: global health politics in a time of economic crises and threatening future trends. Global Health Governance, 2. Disponible en: http://www.ghgj.org

Frenk, J.; Chen, L.; Bhutta, Z.; Cohen, J.; Crisp, N.; Evans, T., Fineberg, H.; Garcia, P.; Ke, Y.; Kelley, P. (2010). Education of Health Professionals for the 21th Century, A Global Independient Comission. Health Professionals for a New Century; transforming education to strengthen health systems in an interdependent world. The Lancet, 376, 1923-1958.

Frenk, J. (1988). La salud pública: campo del conocimiento y ámbito para la acción. Salud Pública, 30, 246-254. México.

Garbossa, G.; Buyayisqui, M. P.; Geffner, L.; López Arias, L. S.; De la Fournière, S.; Haedo, A. S.; Marconi, A.; Frid, J. C.; Nesse, A.; Bordoni, N. (2013). Social and environmental health determinants. Water-and excreta-related parasitoses in a shanty town in Buenos Aires, Argentina. Pathogens and Global Health, 107, $141-152$.

García, R. (2006). Sistemas complejos. Conceptos, método y fundamentación epistemológica de la investigación interdisciplinaria. Barcelona: Gedisa. Gibbons, A,; Limoges, C.; Nowotny, H.; Schwartzman, S.; Scott, P.; Trow, M. (1993). The new production of knowledge: thedynamics of science and reserach in contemporary societies. London: Sage.

Glouberman, S. (2006). Towards dynamic concept of health - CPRN Report. Disponible en: http://www.cprn.org/docs/health/tnp_e.pdf (02/11/2015).

Henry, J. (1990). Meaning Practice in Experiential Learning. Warner Weil, S.; Mc Gill, I. (Eds.), Making Sense of Experiential Learning. Buckingham: The Society for Research into Higher Education \& The Open University.

Inglehart, M.R.; Bagramiam, R.A. (2002). Oral Health-Related Quality of life. Chicago: Quintaessence Pub. Co.

Johnston, D.L. (1995). Education and Research on the Global Environment. Higher Education Policy, 8 (4), 23-29.

Kemmis, S. (1988). Cómo planificar la investigación-acción. Barcelona: Laertes. Knight, J. (2005). An internationalization model: responding to new realities and challenges. En De Witt, Het al. (Eds.), Higher education in Latin America. The International Dimension. The World Bank.

Kolb, D. (1984). Experiential Learning. Englewood Cliffs, NJ: Prentice-Hall. Laframboise, H. L. (1973). Health Policy: breaking the problem down in more manageable segments. Canadian Med. Ass. J., 108, 388-393.

Lalonde, M. (1974). A new perspective on health of Canadians. Ottawa, Ontario. Leal, L. (1995). Extensión Universitaria como acción social y su inclusión en el curriculum de todas las carreras. Informe de investigaciones educativas, IX (número especial), 83-111.
Lemaitre, M.J. (2003). Aseguramiento de la calidad en Chile. Impacto y proyecciones. Primer seminario internacional de Educación superior, Calidad y Acreditación. Bogotá: CORCAS.

Lucarelli, E. (2004). Aprendizaje y práctica profesional en terreno: la formación innovadora en el campo de la salud. En Teoría y práctica en la universidad. La innovación en las aulas (Cap. 4). Buenos Aires: Miño y Dávila Editores.

Menéndez, G. (2013). Institucionalización de la extensión. Conceptualización y dimensiones de la extensión. En Menéndez G. y otros, Integración docencia y extensión. Otra forma de enseñar y aprender. Santa Fe: Ediciones UNL.

Murray, C.J.L., et al. (2012). Health inequality: a global perspective. In Leon, D. \& Walt, G. (Eds.), Poverty, inequality and health. London: Oxford University Press.

Natera Peral, A. (2004). La noción de gobernanza como gestión pública participativa y reticular. Madrid: Universidad Carlos III Ed.

Nowotny, H.; Scott, P.; Gibbons, M. (2001). Rethinking science: knowledge in an age of uncertainty. Cambridge: Polity.

Nuñez, V. (2002). La educación en tiempos de incertidumbre: las apuestas de la Pedagogía Social. Barcelona. Gedisa.

Pagliai, L.; Bordoni, N. (2007). Propuesta de indicadores de impacto social de la investigación en salud pública. Documento para la autoevaluacion de los Sistemas de investigación de países en desarrollo. Revista de la Maestría en Salud Pública, 5 (9, agosto).

Pereyra, L. (2010). Programa de salud bucal en comunidades rurales. Estudio de un caso: Caraguatay, Taruma y Guaraypo, Pcia. de Misiones Argentina. En Bordoni, N.; Escobar, A.; Castillo, R., Odontología Pediátrica. La salud bucal del niño y el adolescente en el mundo actual. Buenos Aires: Ed. Medica Panamericana. Rovere, M. (1999). Redes en salud. Rosario: Instituto de la Salud J. Lazarte. Sacristán, G.; Pérez Gómez, J. (1989). La enseñanza, su teoría y su práctica. Madrid: Akal.

Saltman, R. et al. (2011). Governing Public Hospitals. Reform strategies and the movement towards institutional autonomy. WHO. European Observatory on Health Systems and Policies. Observatory Studies Series 25.

Salzburg Seminar (Feb 14-18 2001). Universities Project Seminar. The Social and Civic Responsibilities of the University.

Souza Casadinho, J. (2013). Dinámica de uso de los agrotóxicos y su relación con la salud socioambiental. En Carrizo, C. y Berger, M. (Comp.), Justicia ambiental. El trabajo interdisciplinario en agrotóxicos y transgénicos. Córdoba: E-book.

Squassi, A. (2010). Clínica odontopediátrica para pacientes con riesgo médico. En Bordoni, N.; Escobar, A.; Castillo Mercado, R., Odontología Pediátrica. La salud bucal del niño en el mundo actual. Buenos Aires: Ed. Médica Panamericana.

Squassi, A.; Cadile, M.; Sotelo, R.; Bordoni, N. (2006). Teacher's perception of oral health. Design, validation and evaluation system. Acta Odont. Latinoamericana, 19 (2), 67-74.

Tedesco, J.C. (1998). Conferencia Inaugural del II Congreso de Educación Social. Madrid.

Van Ginkel, H. (1995). University 2050: The Organization of Creativity and Innovation. Higher Education Policy, 8 (4), 14-20.

Weber, M. (1980). El político y el científico. Madrid: Alianza.

Wiltshire, K. (14 de marzo de 2001). Discurso inaugural. Reunión del Consejo Intergubernamental del Programa Gestión de las Transformaciones Sociales. París. 